\title{
Heavy quarks thermalization in heavy ions ultrarelativistic collisions: Elastic or radiative?
}

\author{
Pol Bernard Gossiaux†§, Vincent Guiho†, and Jörg Aichelin† \\ † SUBATECH, École des Mines de Nantes, 4 rue Alfred Kastler, 44307 Nantes Cedex \\ 3, France
}

\begin{abstract}
We present a dynamical model of heavy quark evolution in the quarkgluon plasma (QGP) based on the Fokker-Planck equation. We then apply this model to the case of ultra-relativistic nucleus-nucleus collisions performed at RHIC in order to investigate which experimental observables might help to discriminate the fundamental process leading to thermalization.
\end{abstract}

\section{Introduction}

The question of the thermalization of heavy quarks produced in ultrarelativistic heavy ions collisions (URHIC) at RHIC and LHC is a fundamental issue because:

- it permits to address the question of the interaction of these quarks with the state of matter\| formed in these collisions and thus hopefully to probe this state of matter.

- the $\mathrm{c}$ and $\overline{\mathrm{c}}$ quarks distributions in the QGP is a mandatory ingredient to describe precisely the $\mathrm{c}+\overline{\mathrm{c}} \rightarrow \psi+X$ processes and to allow a conclusive interpretation of $\psi$ suppression or enhancement $[1,2,3]$.

Although we are strongly convinced that a global analysis of data on open and hidden charm (an beauty) will permit to constrain the models and understand the physics in a more comprehensive way, we will essentially concentrate on the first topic in this contribution.

The recent measurement of the $R_{A A}$ function for non-photonic electrons observed in URHIC clearly indicates that heavy quarks undergo sensitive energy loss in QGP and has lead to various calculations in the frame of perturbative QCD [4] which seem to indicate that radiative energy loss alone cannot cope with the data and that one has to add the contribution from collisional energy loss as well [5]. On the other side, recent work [6] indicates that some aspects of energy loss deduced up to now might be modified once renormalization corrections are included, as for instance the logarithmic growth of

$\S$ To whom correspondence should be addressed (pol.gossiaux@subatech.in2p3.fr)

\| That we will call hereafter "quark gluon plasma" (QGP), with a certain lack of rigor.

ฯ Although it has been neglected for a long time. 
collisional energy loss with the parton energy, what sheds some doubts on the absolute value of both contributions...

Here, we advocate that considering alternate signature as well, like elliptical flow of D mesons (and ensuing decay electrons) or azimuthal correlations could help in order to distinguish the microscopic mechanisms responsible for energy loss and thermalization of heavy quarks. In order to perform this study, we will adopt a transport model for heavy quarks based on the resolution of the Fokker Planck equation in a supposedly equilibrated QGP, with transport (drag and diffusion) coefficients evaluated relying on the microscopic processes - collisional and radiative - but also allowing for some rescaling in order to mock larger or smaller coupling of the heavy quarks with the QGP.

\section{The model}

\subsection{Transport coefficients}

Following [7, 8], the $\mathrm{c}$ and $\overline{\mathrm{c}}$ quarks distributions in the QGP are assumed to follow a Fokker-Planck (FP) equation in momentum space:

$$
\frac{\partial f(\vec{p}, t)}{\partial t}=\frac{\partial}{\partial p_{i}}\left[A_{i}(\vec{p}) f(\vec{p}, t)+\frac{\partial}{\partial p_{j}}\left(B_{i j}(\vec{p}) f(\vec{p}, t)\right)\right]
$$

The main justification for this hypothesis is that the heavy mass of these quarks implies large relaxation times as compared to the typical time of individual $\mathrm{c}+\mathrm{g} \rightarrow \mathrm{c}^{\prime}+\mathrm{g}^{\prime}$ and $\mathrm{c}+\mathrm{q} \rightarrow \mathrm{c}^{\prime}+\mathrm{q}^{\prime}$ collisions, whatever the momentum of the heavy quark. The drag $(A)$ and diffusion $(B)$ coefficients were evaluated according to $[7,8,9]$, resorting to a Kramers-Moyal power expansion of the Boltzmann kernel of $2 \rightarrow 2$ collisions. Notice that the diffusion tensor $B$ admits a transverse - longitudinal decomposition (along the direction of the particle in the frame where the fluid is at rest) and just contains two independent coefficient $B_{\|}$and $B_{\perp}$.
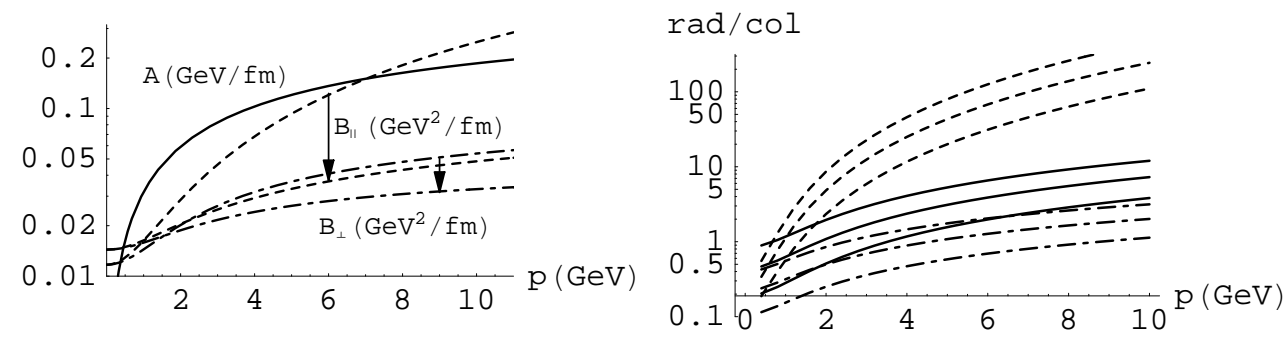

Figure 1. Left: Drag $A$ (plain line) and diffusion $B_{\|}$(dashed) and $B_{\perp}$ (dotted dashed) collisional coefficients for $T=0.25 \mathrm{GeV}$. The arrows indicates the rescaling process performed in order to guarantee the correct asymptotic distribution. Right: ratios of the radiative coefficients with their collisional equivalent (same drawing conventions as left). 
As realized in [10], the asymptotic distribution coming out of the FP evolution deviates from a (relativistic) Maxwell-Boltzmann distribution. This results from the truncation of the Kramers-Moyal series. We have therefore corrected the $B$ coefficients in order to guarantee a correct Maxwell-Boltzmann asymptotic distribution. We have also checked on various examples that this modification has little effect at small evolution time. This constitutes our reference set of "collisional" FP coefficients, as illustrated on figure 1 (left). In order to circumvent our lack of knowledge on the c-QGP interaction (renormalization corrections, non perturbative aspects, etc.), we will also consider alternative sets, obtained by multiplying the reference one by a numerical factor $K_{\text {col }}$.

Coming to the radiative processes, they are expected to dominate at high energy and we have thus consistently followed the Gunion and Bertsch approach and extended it for the case of one massive quark (mass $M$ ). For collisions between two massless quarks leading to the radiation of a gluon carrying a small fraction $x$ of the incoming longitudinal momentum and a transverse $\vec{k}_{\perp}$ component, one has according to [11]:

$$
\mathcal{M}_{q q}^{\mathrm{rad}} \propto \mathcal{M}_{q q}^{\mathrm{col}} \times \epsilon_{\perp} \cdot\left(\frac{\vec{k}_{\perp}}{k_{\perp}^{2}}+\frac{\left(\vec{l}_{\perp}-\vec{k}_{\perp}\right)}{\left(\vec{l}_{\perp}-\vec{k}_{\perp}\right)^{2}}\right),
$$

where $l$ is the momentum transfer in the binary qq collision and the " $\propto$ " refers to some color factor omitted here. In the case of some high energy radiative qQ collision, one shows that the heavy quark has little impact on the radiation emitted in the direction of the light one. On the other side, if the radiation goes along the heavy quark direction, the first term inside the bracket is suppressed by a dead cone factor:

$$
\frac{\vec{k}_{\perp}}{k_{\perp}^{2}} \rightarrow \frac{\vec{k}_{\perp}}{k_{\perp}^{2}+x^{2} M^{2}} .
$$

As for the $g Q \rightarrow Q g g$ radiative mechanism, we have limited ourself to the study of the t-channel, which dominates at high energy and found similar conclusions as for the $q Q \rightarrow Q q g$ process.

Having defined the microscopic radiative process, we evaluated the related transport coefficients for heavy quark along the same line as for the elastic collisions. Typical results are presented in figure 1. Notice that no LPM effect has been taken into effect in our calculation and could then be mocked applying a $K_{\text {rad }}(<1)$ to the coefficients.

It is our hope that the gross experimental results will ultimately permit to fix approximate values of $K_{\mathrm{col}}$ and $K_{\mathrm{rad}}$ and that our model could then be used to predict even finer aspects. In this respect, we view the model as a semi-predictive effective theory that could be useful to match the gap between the fundamental underlying theory (QCD) and experimental results.

\subsection{Other Ingredients}

The FP coefficients depend on position and time (only) through the local temperature and velocity of the surrounding medium, that is assumed to be locally thermalized and described via hydrodynamic evolution. Therefore, we do need to evaluate explicitly all 
microscopic $\mathrm{c}+\mathrm{g} \rightarrow \mathrm{c}^{\prime}+\mathrm{g}^{\prime}$ and $\mathrm{c}+\mathrm{q} \rightarrow \mathrm{c}^{\prime}+\mathrm{q}^{\prime}$ processes when performing our simulations: only c, $\overline{\mathrm{c}}$ and $J / \psi$ d.o.f. are considered.

Once the $\mathrm{c}$ and $\overline{\mathrm{c}}$ quarks reach the boundary of the QGP phase, we convert them into D and $\bar{D}$ mesons either via fragmentation (using Peterson's fragmentation function) or via coalescence (with the light quarks sampled out from the thermal bath), or via some mixed process (mainly fragmentation for fast $\mathrm{c}$ and coalescence for slow ones). In fact, we have observed that most of our results merely depend on the precise mechanism chosen to convert c quarks into mesons and we will therefore not pay too much attention to this aspect here.

For the sake of simplicity, we have neglected all initial state interactions. We have also allowed ourselves to take a simple factorized form of the initial $\mathrm{c}-\overline{\mathrm{c}}$ phasedistribution, i.e. $f_{\text {in }}\left(\vec{r}_{\mathrm{c}}, \vec{p}_{\mathrm{c}} ; \vec{r}_{\overline{\mathrm{c}}}, \vec{p}_{\overline{\mathrm{c}}}\right) \propto T_{A}\left(\vec{r}_{\mathrm{c}, \perp}\right) T_{B}\left(\vec{r}_{\overline{\mathrm{c}}, \perp}\right) \delta^{(3)}\left(\vec{r}_{\mathrm{c}}-\vec{r}_{\overline{\mathrm{c}}}\right) \delta\left(z_{\mathrm{c}}\right) \times f_{\text {in }}\left(\vec{p}_{\mathrm{c}}\right) \times f_{\text {in }}\left(\vec{p}_{\overline{\mathrm{c}}}\right)$ with $f_{\text {in }}\left(\vec{p}_{\mathrm{c}}\right) \propto f_{\text {in }}\left(\vec{p}_{\mathrm{c}, \perp}\right) \times f_{\text {in }}\left(y_{c}\right)$, where $f_{\text {in }}\left(y_{\mathrm{c}}\right)$ has been chosen according to [12] and $f_{\text {in }}\left(\vec{p}_{\mathrm{c}, \perp}\right)$ in order to reproduce the $\mathrm{D}$ transverse-momentum spectra at mid-rapidity in nucleon-nucleon reactions, once the fragmentation mechanism is included. No b quarks have been included in the present work.

\section{Results and discussion}

All results are for $\mathrm{Au}+\mathrm{Au}$ reactions at $\sqrt{s}=200 \mathrm{GeV}$. The initial number $N_{\mathrm{c}}$ of $\mathrm{c}$ and $\overline{\mathrm{c}}$ quarks is taken to be 40 , with $\frac{\mathrm{d} N_{\mathrm{c}}(y=0)}{\mathrm{d} y} \approx 9$. Unless specified differently, the temperature and velocity profiles have been evaluated with the hydrodynamic model of Kolb and Heinz [13].
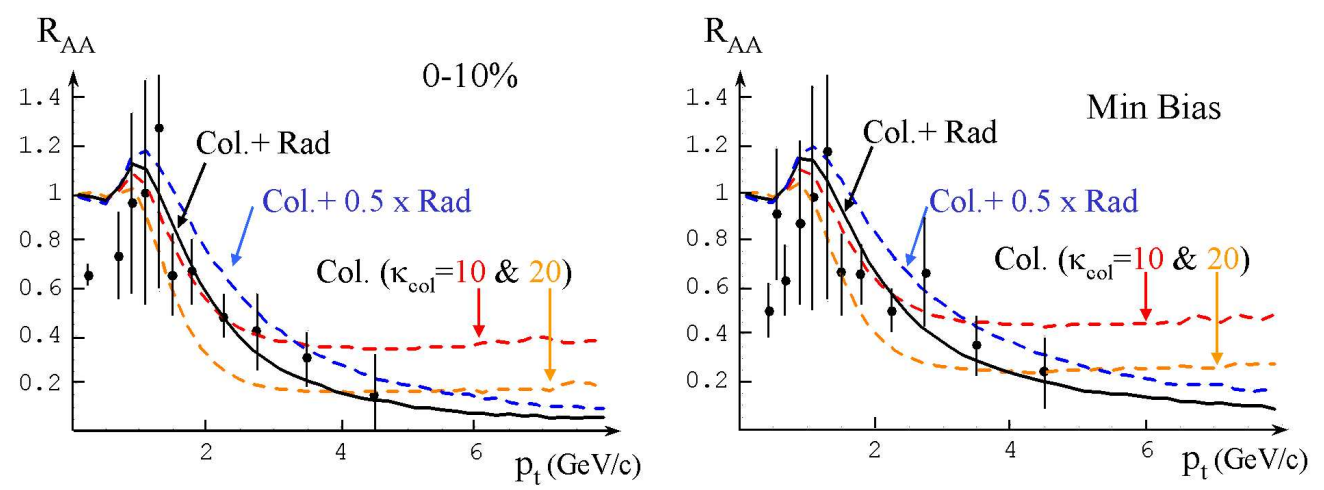

Figure 2. $R_{A A}$ for most central collisions (left) or minimum biased data (right). Data are form the PHENIX experiment.

We have first "calibrated" our transport coefficients ${ }^{+}$resorting to the $R_{A A}$ function for (non photonic) single electrons, for various centralities. As illustrated on fig. 2,

+ Results presented in this preliminary study of the "radiative" FP coefficients were in fact obtained without including the dead cone effect. Although the main conclusions are expected to be robust against this fact, precise results and favoured value of $K_{\text {rad }}$ will probably depend on it. 
collisional processes have to be cranked up by a factor 10 - 20 (set I) in order to reproduce the (PHENIX) data. On the other side, including both collisional and radiative processes (set II), even including a $K_{\text {rad }}=0.5$ in order to mock some LPM effect, provides a good agreement with the data all over their range. Although one would have a natural tendency to discard the first set and just consider the second one, we will pursue the analysis with both in order to investigate how well one can distinguish processes with other experimental observables.

We now turn to the analysis of elliptical flow. The most striking features - cf. fig 3 - are as follows:

- The comparison between left and right panels indicates that elliptical flows associated to both sets differ and thus that one could distinguish the various mechanisms responsible for heavy quark energy loss and thermalization by the joint analysis of $R_{A A}$ and $v_{2}$.

- However, the absolute amplitude of the elliptical flow obtained in PHENIX experiment cannot be reproduced even in the most favorable case - set I -, what could indicate that a significant part of the $v_{2}$ of $\mathrm{D}$ mesons is achieved within the hadronic phase (not implemented in our simulations).

- The rather moderate $v_{2}$ obtained within our calculations has other origins like the lack of total thermalization for the heavy quarks and the small contribution from the light quarks to the $v_{2}$ of $\mathrm{D}$ mesons after coalescence.
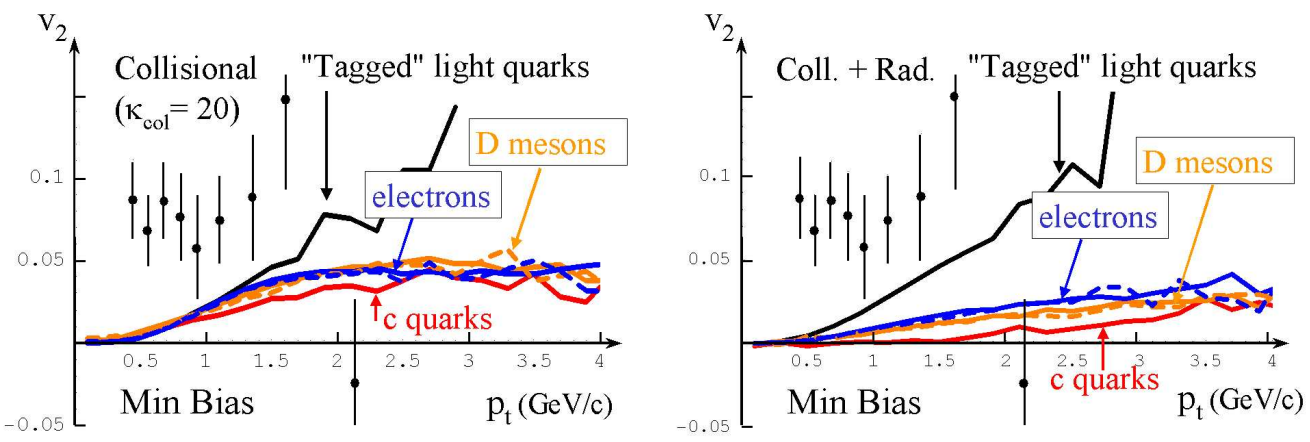

Figure 3. Left: elliptical flow of c quarks (as they leave the QGP), D mesons (plain curve for coalescence mechanism and dashed curve for Peterson fragmentation) and ensuing decay electrons for the set I of transport coefficients. Also shown is the elliptical flow of the "tagged" light quarks (see text). Right: same quantities for set II (radiative + collisional).

- Apart from the well-known fact that quarks which coalesce to form a D mesons must have roughly the same velocity, what implies a much lower momentum and thus $v_{2}$ for the light one, another part of the explanation might be that the heavy quarks are preferably produced though hard reactions in the middle of the transverse zone 
and do not encounter the fluid cells baring a large $v_{2}$. This is demonstrated on fig. 3 by the moderate $v_{2}$ of the so-called "tagged" quarks, i.e. light quarks sampled out of the hydrodynamical cells at the points where the c quarks escape from the QGP.

We conclude our study with the analysis of azimuthal correlations of $D-\bar{D}$ pairs. Although it is a rather crude approximation, we will assume, for this specific observable, that the $\mathrm{c}$ and $\overline{\mathrm{c}}$ quarks are produced back to back in the original nucleon-nucleon reaction, in order to better appreciate how their interactions with the QGP affects these correlations. We also have limited the analysis to $\mathrm{D}$ and $\overline{\mathrm{D}}$ of average $p_{t}$ produced at mid-rapidity.

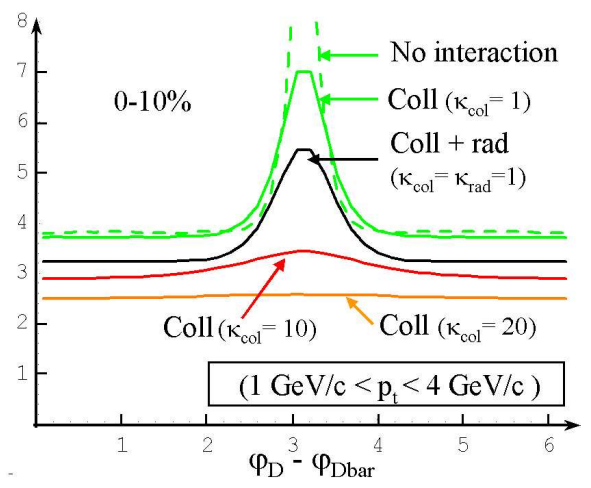

Figure 4. Azimuthal correlations of $\mathrm{D}-\overline{\mathrm{D}}$ pairs produced in $\mathrm{Au}-\mathrm{Au}$ central collisions. Various sets of transport coefficients have been used (cf. text).

On fig. 4, one observes some smearing of the azimuthal correlation even without any interaction with the QGP, a coincidence effect due to the numerous c $\bar{c}$ pairs created in the URHIC. Coming to the impact of these interactions, one observes a clear disappearance of the correlation with increasing $K_{\text {col }}$ (for purely elastic processes), while the correlation patterns stemming from set I and set II are clearly distinguishable. Therefore, it appears that joint analysis of $R_{A A}$ and azimuthal correlations could permit as well to discriminate better the mechanisms at hand for energy loss of heavy quarks.

\section{Conclusion and outlook}

We have presented a model that copes efficiently with dynamical evolution of heavy quarks in QGP, based on the Fokker Planck equation. We have exhibited two sets of transport coefficients which permit to reproduce the $R_{A A}$ of non photonic electrons at RHIC. We have then shown that other observables, like elliptical flow of $\mathrm{D}$ and $\overline{\mathrm{D}}$ mesons or azimuthal correlations of $\mathrm{D}-\overline{\mathrm{D}}$ pairs are sensitive to the microscopic mechanism (elastic or radiative) and could then help in order to discriminate between them. However, we underpredict the experimental values of the elliptical flow of single 
electrons whatever the set chosen. Several hypothesis could explain this fact - the most critical being the failure of the Fokker-Planck approach to describe heavy quark evolution in QGP - and deserve further investigation.

\section{References}

[1] Braun-Munziger P and Stachel J 2000, Phys. Lett. B 490196

[2] Thews R L, Schroedter M and Rafelski J 2001 Phys. Rev. C 63054905

[3] Gossiaux P-B, Guiho V and Aichelin J 2005 Journal of Physics G: Nucl. Part. Phys. 31 S1079

[4] Djordjevic M, Gyulassy M, Vogt R and Wicks S 2006 Phys. Lett. B 63281

[5] Wicks S, Horowitz W, Djordjevic M and Gyulassy M 2005 Preprint nucl-th/0512076

[6] Peshier A 2006 Preprint hep-ph/0605294

[7] Svetitsky B 1988 Phys. Rev. D 372484

[8] Mustafa M G, Pal D and Srivastava D K 1998 Phys. Rev. C 57889

[9] Cleymans J and Ray P S 1985 BI-TP 85/08

[10] Walton D B and Rafelski J 2000 Phys. Rev. Lett. 84 31-34

[11] Gunion J F and Bertsch G 1982 Phys. Rev. D 25746

[12] Vogt R 2003 Int.J.Mod.Phys. E 12 211-270

[13] Kolb P F and Heinz U 2003 Quark Gluon Plasma 3 (Hwa R C and Wang X N, World Scientific, Singapore) 634 